\title{
CAFFARELli-KoHN-NiREnBERG INEQUALITY ON METRIC MEASURE SPACES WITH APPLICATIONS
}

\author{
Alexandru Kristály \\ Department of Economics, Babeş-Bolyai University, 400591 Cluj-Napoca, Romania \\ Email addresses: alexandrukristaly@yahoo.com; alexandru.kristaly@econ.ubbcluj.ro \\ Shin-ichi Ohta \\ Department of Mathematics, Kyoto University, Kyoto 606-8502, Japan \\ Email address: sohta@math.kyoto-u.ac.jp \\ Dedicated to Professor Vicenţiu Rădulescu on the occasion of his $55^{\text {th }}$ birthday
}

\begin{abstract}
We prove that if a metric measure space satisfies the volume doubling condition and the Caffarelli-Kohn-Nirenberg inequality with the same exponent $n \geq 3$, then it has exactly the $n$-dimensional volume growth. As an application, if an $n$-dimensional Finsler manifold of non-negative $n$-Ricci curvature satisfies the Caffarelli-KohnNirenberg inequality with the sharp constant, then its flag curvature is identically zero. In the particular case of Berwald spaces, such a space is necessarily isometric to a Minkowski space.
\end{abstract}

Keywords: Caffarelli-Kohn-Nirenberg inequality; metric measure spaces; volume doubling condition; Finsler manifolds.

$M S C: 35 \mathrm{R} 06,53 \mathrm{C} 60,58 \mathrm{~J} 60$.

\section{Introduction and statement of main results}

Let $a \in[0,1)$ be a parameter, $n \geq 3$ be an integer, and put $p=2 n /(n-2+2 a)$. In the theory of Sobolev inequalities, a central role is played by the famous Caffarelli-KohnNirenberg inequality (see [4]) which states that

$$
\left(\int_{\mathbb{R}^{n}} \frac{|u|^{p}}{|x|^{a p}} d x\right)^{\frac{1}{p}} \leq K_{a}\left(\int_{\mathbb{R}^{n}}|D u|^{2} d x\right)^{\frac{1}{2}} \quad \text { for all } u \in C_{0}^{\infty}\left(\mathbb{R}^{n}\right),
$$

where

$$
K_{a}:=\left(\frac{1}{(n-2)(n-a p)}\right)^{\frac{1}{2}}\left(\frac{(2-a p) \Gamma((2 n-2 a p) /(2-a p))}{n \omega_{n} \Gamma^{2}((n-a p) /(2-a p))}\right)^{\frac{2-a p}{2 n-2 a p}}
$$

is the optimal constant (see Lieb [9]), $\omega_{n}:=\pi^{n / 2} / \Gamma(n / 2+1)$ being the volume of the unit ball in $\mathbb{R}^{n}$. Moreover, a family of extremals is given by

$$
u_{\lambda}(x)=\left(\lambda+|x|^{2-a p}\right)^{\frac{2-n}{2-a p}}, \quad \lambda>0 .
$$


The optimal constant and extremals for $a=0$ have been established by Aubin [2] and Talenti [17] in which case the above inequality reduces to the standard Sobolev inequality; see Chou and Chu [5] for the most general case. Furthermore, various versions of the Caffarelli-Kohn-Nirenberg inequality have been treated also on Riemannian manifolds and Orlicz-Sobolev spaces (see, e.g., do Carmo and Xia [6]).

The main objective of the present paper is to investigate the Caffarelli-Kohn-Nirenberg inequality in the context of metric measure spaces. As applications, we provide novel rigidity results for Finsler manifolds by means of the sharp Caffarelli-Kohn-Nirenberg inequality.

In order to state the main result of the paper, we fix the numbers $a, n$ and $p$ as above. Let $(X, d)$ be a metric space and $\mu$ be a Borel measure on $X$ such that $0<\mu(U)<\infty$ for any nonempty bounded open set $U \subset X$. For some element $x_{0} \in X$ and constant $C>0$, we consider the Caffarelli-Kohn-Nirenberg inequality on $(X, d, \mu)$ of the form

$$
\left(\int_{X} \frac{|u(x)|^{p}}{d\left(x_{0}, x\right)^{a p}} d \mu(x)\right)^{\frac{1}{p}} \leq C\left(\int_{X}|D u|(x)^{2} d \mu(x)\right)^{\frac{1}{2}} \text { for all } u \in \operatorname{Lip}_{0}(X) . \quad(\mathbf{C K N})_{C}^{x_{0}}
$$

Hereafter, $\operatorname{Lip}_{0}(X)$ is the space of Lipschitz functions with compact support on $X$, while

$$
|D u|(x):=\limsup _{y \rightarrow x} \frac{|u(y)-u(x)|}{d(x, y)}
$$

is the local Lipschitz constant of $u$ at $x \in X$. The function $x \longmapsto|D u|(x)$ is Borel measurable for $u \in \operatorname{Lip}_{0}(X)$. For instance, any bi-Lipschitz deformation of the Euclidean space $\mathbb{R}^{n}$ satisfies $(\mathbf{C K N})_{C}^{x_{0}}$ with some $C \geq K_{a}$.

For some fixed elements $C_{0} \geq 1$ and $x_{0} \in X$, we introduce the following hypotheses on the behavior of $\mu$ :

$(\mathbf{V D})_{C_{0}}^{n} \frac{\mu(B(x, R))}{\mu(B(x, r))} \leq C_{0}\left(\frac{R}{r}\right)^{n}$ for all $x \in X$ and $0<r<R$;

$(\mathbf{A R})_{x_{0}}^{n} \liminf _{r \rightarrow 0} \frac{\mu\left(B\left(x_{0}, r\right)\right)}{\mu_{E}\left(\mathbb{B}_{n}(r)\right)}=1$.

As usual, $B(x, r):=\{y \in X: d(x, y)<r\}, \mathbb{B}_{n}(r):=\left\{x \in \mathbb{R}^{n}:|x|<r\right\}$, and $\mu_{E}$ is the $n$-dimensional Lebesgue measure.

The main result of the paper can be stated as follows.

Theorem 1.1 Let $a \in[0,1), n \geq 3, p=2 n /(n-2+2 a), x_{0} \in X, C \geq K_{a}$, and $C_{0} \geq 1$. Assume that the Caffarelli-Kohn-Nirenberg inequality $(\mathbf{C K N})_{C}^{x_{0}}$ holds on a proper metric measure space $(X, d, \mu)$, and the hypotheses $(\mathbf{V D})_{C_{0}}^{n}$ and $(\mathbf{A R})_{x_{0}}^{n}$ are verified. Then, for every $x \in X$ and $\rho>0$, we have

$$
\mu(B(x, \rho)) \geq C_{0}^{-1}\left(C^{-1} K_{a}\right)^{\frac{n}{1-a}} \mu_{E}\left(\mathbb{B}_{n}(\rho)\right) .
$$

In particular, $(X, d, \mu)$ has the $n$-dimensional volume growth

$$
C_{0}^{-1}\left(C^{-1} K_{a}\right)^{\frac{n}{1-a}} \omega_{n} \rho^{n} \leq \mu\left(B\left(x_{0}, \rho\right)\right) \leq C_{0} \omega_{n} \rho^{n} \quad \text { for all } \rho>0 .
$$


This theorem extends do Carmo and Xia's result [6. Theorem 1.1] on Riemannian manifolds of non-negative Ricci curvature in two respects. Theorem 1.1 is concerned with general metric measure spaces, and assumes only the volume growth condition (VD) ${ }_{C_{0}}^{n}$ instead of the curvature bound. Before discussing applications, let us give several remarks on the hypotheses and the conclusions of the theorem.

Remark 1.2 (a) We remark that $(\mathbf{C K N})_{C}^{x_{0}}$ ensures that $(X, d)$ is unbounded (equivalently, non-compact). Indeed, if $(X, d)$ is bounded, then $u+c$ with $c \rightarrow \infty$ violates the validity of $(\mathbf{C K N})_{C}^{x_{0}}$.

(b) If $(X, d, \mu)$ satisfies the volume doubling condition:

$$
\mu(B(x, 2 r)) \leq \Lambda \mu(B(x, r)) \text { for some } \Lambda \geq 1 \text { and all } x \in X, r>0,
$$

then we easily see that $(\mathbf{V D})_{C_{0}}^{n}$ is satisfied (with, e.g., $n \geq \log _{2} \Lambda$ and $C_{0}=\Lambda$ ). Thus $(\mathrm{VD})_{C_{0}}^{n}$ can be interpreted as the volume doubling condition with the explicit exponent $n$. One can also regard (VD) ${ }_{C_{0}}^{n}$ as a generalization of the Bishop-Gromov volume growth estimate (of non-negative Ricci curvature).

(c) Note that, on the one hand, (VD) $C_{C_{0}}^{n}$ implies that the Hausdorff dimension $\operatorname{dim}_{H} X$ of $(X, d)$ is at most $n$. On the other hand, since $\lim _{\sup _{r \rightarrow 0}} \mu\left(B\left(x_{0}, r\right)\right) / \mu_{E}\left(\mathbb{B}_{n}(r)\right) \leq C_{0}$ by $(\mathbf{V D})_{C_{0}}^{n}$ and $(\mathbf{A R})_{x_{0}}^{n}$, we have the Ahlfors $n$-regularity at $x_{0}$ in the sense that $\Omega^{-1} r^{n} \leq$ $\mu\left(B\left(x_{0}, r\right)\right) \leq \Omega r^{n}$ for some $\Omega \geq 1$ and small $r>0$, thus we have $\operatorname{dim}_{H} X=n$. (See [7] for the importance of the volume doubling condition and the Ahlfors regularity in analysis on metric measure spaces.) We also remark that the constant 1 was chosen as the RHS of $(\mathbf{A R})_{x_{0}}^{n}$ merely for simplicity. Since $\Omega_{x_{0}}:=\liminf _{r \rightarrow 0} \mu\left(B\left(x_{0}, r\right)\right) / r^{n}$ is positive by $(\mathbf{V D})_{C_{0}}^{n}$, one can normalize $\mu$ so as to satisfy $(\mathbf{A R})_{x_{0}}^{n}$ whenever $\Omega_{x_{0}}$ is bounded.

(d) The volume growth estimate (1.3) shows that, for instance, the cylinder $\mathbb{S}^{n-1} \times \mathbb{R}$ can not support $(\mathbf{C K N})_{C}^{x}$ for any $x$ and $C$.

(e) The use of the measure $\mu_{E}$ as a comparing one to $\mu$ in the hypotheses $(\mathbf{V D})_{C_{0}}^{n}$ and $(\mathbf{A R})_{x_{0}}^{n}$ comes from the fact that the number $K_{a}$ is optimal and the functions from (1.2) are minimizers in the Euclidean Caffarelli-Kohn-Nirenberg inequality. Therefore, if a sharp Caffarelli-Kohn-Nirenberg inequality holds in a generic metric measure space $\left(X_{0}, d_{0}, \mu_{0}\right)$, knowing the optimal constant $K_{0}>0$ and assuming that the class of extremals is formally the same as (1.2) with $d_{0}\left(x_{0}, x\right)$ instead of $|x|$, then one can prove a similar statement to Theorem 1.1 by replacing $\mu_{E}$ and $K_{a}$ with $\mu_{0}$ and $K_{0}$, respectively.

We point out that, on (absolutely homogeneous for simplicity) Finsler manifolds with non-negative $n$-Ricci curvature, $(\mathbf{V D})_{C_{0}}^{n}$ holds with $C_{0}=1$ (see Shen [14], Ohta [10] and Theorem 3.3 below). In particular, from Theorem 1.1, important rigidity results can be deduced in the context of Finsler manifolds when the sharp Caffarelli-Kohn-Nirenberg inequality holds (for precise notions, see Section 3). We state two such results.

Theorem 1.3 Let $a \in[0,1), n \geq 3, p=2 n /(n-2+2 a)$, and $(M, F)$ be a complete $n$ dimensional Finsler manifold. Fix a positive smooth measure $\mu$ on $M$ and assume that the $n$-Ricci curvature $\operatorname{Ric}_{n}$ of $(M, F, \mu)$ is non-negative, the sharp Caffarelli-Kohn-Nirenberg inequality $(\mathbf{C K N})_{K_{a}}^{x_{0}}$ holds for some $x_{0} \in M$, and in addition $\lim _{r \rightarrow 0} \mu\left(B\left(x_{0}, r\right)\right) / \omega_{n} r^{n}=$ 1. Then the flag curvature of $(M, F)$ is identically zero. 
Theorem 1.4 Let $a \in[0,1), n \geq 3, p=2 n /(n-2+2 a)$, and $(M, F)$ be a complete $n$-dimensional Berwald space with non-negative Ricci curvature. If for some $x_{0} \in M$ and the n-dimensional Hausdorff measure of $(M, F)$ the sharp Caffarelli-Kohn-Nirenberg inequality $(\mathbf{C K N})_{K_{a}}^{x_{0}}$ holds, then $(M, F)$ is isometric to a Minkowski space.

Remark 1.5 (a) By using anisotropic symmetrization arguments, we prove in Section 3 that the sharp Caffarelli-Kohn-Nirenberg inequality $(\mathbf{C K N})_{K_{a}}^{x_{0}}$ holds on every Minkowski space $\left(\mathbb{R}^{n}, F\right)$ (Proposition 3.4). In this manner, Theorem 1.4 delimits Minkowski spaces as the optimal geometric framework where $(\mathbf{C K N})_{K_{a}}^{x_{0}}$ holds within the class of Berwald spaces with non-negative Ricci curvature.

(b) Riemannian manifolds and (locally) Minkowski spaces are Berwald spaces. Therefore Theorem 1.4 extends do Carmo and Xia's result [6, Corollary 1.2] in the Riemannian context. In fact, some constructions in the proof of Theorem 1.1 are inspired from [6].

(c) The assumption $n \geq 3$ in Theorem 1.4 is essential not only in the definition of $p=2 n /(n-2+2 a)$ but also for the structure of the Berwald space. Indeed, Szabó's rigidity result states that any Berwald surface is either a locally Minkowski space or a Riemannian surface, see Szabó [16] and Bao, Chern and Shen [3, Theorem 10.6.2].

The paper is constructed as follows. In Section 2, we prove Theorem 1.1. In Section 3, we first recall some basic notions and results from Finsler geometry, and then complete the proof of Theorems 1.3 and 1.4 .

\section{Proof of Theorem 1.1}

We divide the proof into five steps.

Step 1 We first derive an important ODE from the extremals (1.2) in the Euclidean case. Since $u_{\lambda}(x)=\left(\lambda+|x|^{2-a p}\right)^{\frac{2-n}{2-a p}}$ is a minimizer in the Euclidean Caffarelli-Kohn-Nirenberg inequality $(\mathbf{C K N})_{K_{a}}^{x_{0}}$, the following integral identity holds for every $\lambda>0$ :

$$
\left(\int_{\mathbb{R}^{n}} \frac{\left(\lambda+|x|^{2-a p}\right)^{\frac{(2-n) p}{2-a p}}}{|x|^{a p}} d \mu_{E}(x)\right)^{\frac{2}{p}}=K_{a}^{2}(n-2)^{2} \int_{\mathbb{R}^{n}} \frac{\left(\lambda+|x|^{2-a p}\right)^{\frac{2(a p-n)}{2-a p}}}{|x|^{2 a p-2}} d \mu_{E}(x) .
$$

Observe that $\frac{(2-n) p}{2-a p}=\frac{2(a p-n)}{2-a p}=\frac{n}{a-1}<0$, in particular, $a p<2$. We introduce the auxiliary function $Q_{E}:(0, \infty) \longrightarrow \mathbb{R}$ defined by

$$
Q_{E}(\lambda):=\frac{1-a}{n-1+a} \int_{\mathbb{R}^{n}} \frac{\left(\lambda+|x|^{2-a p}\right)^{\frac{n-1+a}{a-1}}}{|x|^{a p}} d \mu_{E}(x) .
$$

Then the identity (2.1) reduces to, provided that $Q_{E}$ is well-defined,

$$
\left(-Q_{E}^{\prime}(\lambda)\right)^{\frac{2}{p}}=K_{a}^{2}(n-2)^{2}\left(\frac{n-1+a}{1-a} Q_{E}(\lambda)+\lambda Q_{E}^{\prime}(\lambda)\right), \quad \lambda>0 .
$$


To see that $Q_{E}$ is well-defined, we obtain from the layer cake representation of functions and a change of variables as $t=\left(\lambda+\rho^{2-a p}\right)^{\frac{n-1+a}{a-1}} \rho^{-a p}$ that

$$
\begin{aligned}
Q_{E}(\lambda) & =\frac{1-a}{n-1+a} \int_{0}^{\infty} \mu_{E}\left\{x \in \mathbb{R}^{n}: \frac{\left(\lambda+|x|^{2-a p}\right)^{\frac{n-1+a}{a-1}}}{|x|^{a p}}>t\right\} d t \\
& =\frac{1-a}{n-1+a} \int_{0}^{\infty} \mu_{E}\left\{x \in \mathbb{R}^{n}:|x|<\rho\right\} f(\lambda, \rho) d \rho,
\end{aligned}
$$

where $f:(0, \infty)^{2} \longrightarrow \mathbb{R}$ is given by

$$
\begin{aligned}
f(\lambda, \rho) & =\frac{n-1+a}{1-a} \frac{\left(\lambda+\rho^{2-a p}\right)^{\frac{n}{a-1}}}{\rho^{a p}}(2-a p) \rho^{1-a p}+a p \frac{\left(\lambda+\rho^{2-a p}\right)^{\frac{n-1+a}{a-1}}}{\rho^{a p+1}} \\
& =\frac{\left(\lambda+\rho^{2-a p}\right)^{\frac{n}{a-1}}}{\rho^{a p+1}}\left\{\rho^{2-a p}\left(\frac{n-1+a}{1-a}(2-a p)+a p\right)+a p \lambda\right\} .
\end{aligned}
$$

Hence we have

$$
Q_{E}(\lambda)=\frac{1-a}{n-1+a} \int_{0}^{\infty} \mu_{E}\left(\mathbb{B}_{n}(\rho)\right) f(\lambda, \rho) d \rho .
$$

An elementary calculus shows that the improper integral in (2.4) converges, thus $Q_{E}$ is well-defined.

Step 2 Switching to the metric measure setting as in Theorem 1.1, we first observe that the hypotheses $(\mathbf{V D})_{C_{0}}^{n}$ and $(\mathbf{A R})_{x_{0}}^{n}$ yield

$$
\mu\left(B\left(x_{0}, \rho\right)\right) \leq C_{0} \mu_{E}\left(\mathbb{B}_{n}(\rho)\right) \text { for every } \rho>0 .
$$

Let us consider for each $\lambda>0$ the sequence of functions $u_{\lambda, k}: X \longrightarrow \mathbb{R}, k \in \mathbb{N}$, defined by

$$
u_{\lambda, k}(x):=\max \left\{0, \min \left\{0, k-d\left(x_{0}, x\right)\right\}+1\right\}\left(\lambda+\max \left\{d\left(x_{0}, x\right), k^{-1}\right\}^{2-a p}\right)^{\frac{2-n}{2-a p}} .
$$

Since $(X, d)$ is proper, $\operatorname{supp}\left(u_{\lambda, k}\right)=\left\{x \in X: d\left(x_{0}, x\right) \leq k+1\right\}$ is compact. Therefore we have $u_{\lambda, k} \in \operatorname{Lip}_{0}(X)$ for every $\lambda>0$ and $k \in \mathbb{N}$. We set

$$
\tilde{u}_{\lambda}(x):=\lim _{k \rightarrow \infty} u_{\lambda, k}(x)=\left(\lambda+d\left(x_{0}, x\right)^{2-a p}\right)^{\frac{2-n}{2-a p}} .
$$

Since the functions $u_{\lambda, k}$ verify the Caffarelli-Kohn-Nirenberg inequality $(\mathbf{C K N})_{C}^{x_{0}}$, a simple approximation procedure based on (2.5) shows that $\tilde{u}_{\lambda}$ verifies $(\mathbf{C K N})_{C}^{x_{0}}$ as well. Consequently, we can apply $(\mathbf{C K N})_{C}^{x_{0}}$ to $\tilde{u}_{\lambda}$. In particular, by exploiting a chain rule for the local Lipschitz constant and the fact that $x \longmapsto d\left(x_{0}, x\right)$ is 1-Lipschitz (thus $\left|\operatorname{Dd}\left(x_{0}, \cdot\right)\right|(x) \leq 1$ for all $\left.x\right)$, we obtain

$$
\left(\int_{X} \frac{\left(\lambda+d\left(x_{0}, x\right)^{2-a p}\right)^{\frac{(2-n) p}{2-a p}}}{d\left(x_{0}, x\right)^{a p}} d \mu(x)\right)^{\frac{2}{p}} \leq C^{2}(n-2)^{2} \int_{X} \frac{\left(\lambda+d\left(x_{0}, x\right)^{2-a p}\right)^{\frac{2(a p-n)}{2-a p}}}{d\left(x_{0}, x\right)^{2 a p-2}} d \mu(x) .
$$


We shall rewrite (2.6) by means of the function $\tilde{Q}:(0, \infty) \longrightarrow \mathbb{R}$ defined by

$$
\tilde{Q}(\lambda):=\frac{1-a}{n-1+a} \int_{X} \frac{\left(\lambda+d\left(x_{0}, x\right)^{2-a p}\right)^{\frac{n-1+a}{a-1}}}{d\left(x_{0}, x\right)^{a p}} d \mu(x) .
$$

Before to do that, we claim that $\tilde{Q}$ is well-defined. Again, by the layer cake representation of functions, one has

$$
\tilde{Q}(\lambda)=\frac{1-a}{n-1+a} \int_{0}^{\infty} \mu\left\{x \in X: \frac{\left(\lambda+d\left(x_{0}, x\right)^{2-a p}\right)^{\frac{n-1+a}{a-1}}}{d\left(x_{0}, x\right)^{a p}}>t\right\} d t .
$$

By taking into account that $\operatorname{diam} X=\infty$, similarly to the previous step, we have

$$
\tilde{Q}(\lambda)=\frac{1-a}{n-1+a} \int_{0}^{\infty} \mu\left(B\left(x_{0}, \rho\right)\right) f(\lambda, \rho) d \rho .
$$

In particular, from (2.5) and (2.4), for every $\lambda>0$ we obtain

$$
0<\tilde{Q}(\lambda) \leq \frac{C_{0}(1-a)}{n-1+a} \int_{0}^{\infty} \mu_{E}\left(\mathbb{B}_{n}(\rho)\right) f(\lambda, \rho) d \rho=C_{0} Q_{E}(\lambda),
$$

which concludes the claim. Now, similarly to (2.2), we can transform the relation (2.6) via $\tilde{Q}$ into the inequality

$$
\left(-\tilde{Q}^{\prime}(\lambda)\right)^{\frac{2}{p}} \leq C^{2}(n-2)^{2}\left(\frac{n-1+a}{1-a} \tilde{Q}(\lambda)+\lambda \tilde{Q}^{\prime}(\lambda)\right), \quad \lambda>0 .
$$

Inspired from (2.2) and (2.8), we consider the ODE

$$
\left(-q^{\prime}(\lambda)\right)^{\frac{2}{p}}=C^{2}(n-2)^{2}\left(\frac{n-1+a}{1-a} q(\lambda)+\lambda q^{\prime}(\lambda)\right), \quad \lambda>0 .
$$

On account of (2.2), one can observe that (2.9) has the particular solution of the form

$$
q(\lambda)=\left(C^{-1} K_{a}\right)^{\frac{n}{1-a}} Q_{E}(\lambda) .
$$

Step 3 We shall show that, for every $\lambda>0$,

$$
\tilde{Q}(\lambda) \geq q(\lambda)
$$

Suppose $C>K_{a}$ without loss of generality. The proof of (2.10) requires a local (near zero) and a global treatment of the quotient $\tilde{Q} / q$. First, due to the hypothesis $(\mathbf{A R})_{x_{0}}^{n}$, for every $\varepsilon>0$ there exists $\rho_{\varepsilon}>0$ such that $\mu\left(B\left(x_{0}, \rho\right)\right) \geq(1-\varepsilon) \mu_{E}\left(\mathbb{B}_{n}(\rho)\right)$ for all $\rho \in\left[0, \rho_{\varepsilon}\right]$. Therefore, by (2.7) and changing the variables as $\rho=\lambda^{\frac{1}{2-a p}} t$, it turns out that

$$
\begin{aligned}
\tilde{Q}(\lambda) & \geq \frac{1-a}{n-1+a}(1-\varepsilon) \int_{0}^{\rho_{\varepsilon}} \mu_{E}\left(\mathbb{B}_{n}(\rho)\right) f(\lambda, \rho) d \rho \\
& =\frac{1-a}{n-1+a}(1-\varepsilon) \lambda^{\frac{n-2+2 a}{2(a-1)}} \int_{0}^{\rho_{\varepsilon} \lambda^{\frac{1}{a p-2}}} \mu_{E}\left(\mathbb{B}_{n}(t)\right) f(1, t) d t .
\end{aligned}
$$


A similar argument gives from (2.4) that

$$
Q_{E}(\lambda)=\frac{1-a}{n-1+a} \lambda^{\frac{n-2+2 a}{2(a-1)}} \int_{0}^{\infty} \mu_{E}\left(\mathbb{B}_{n}(t)\right) f(1, t) d t
$$

The above relations and the fact $a p-2<0$ lead to

$$
\liminf _{\lambda \rightarrow 0} \frac{\tilde{Q}(\lambda)}{q(\lambda)}=\left(C K_{a}^{-1}\right)^{\frac{n}{1-a}} \liminf _{\lambda \rightarrow 0} \frac{\tilde{Q}(\lambda)}{Q_{E}(\lambda)} \geq\left(C K_{a}^{-1}\right)^{\frac{n}{1-a}}(1-\varepsilon) .
$$

Since $\varepsilon>0$ is arbitrarily small, we obtain

$$
\liminf _{\lambda \rightarrow 0} \frac{\tilde{Q}(\lambda)}{q(\lambda)} \geq\left(C K_{a}^{-1}\right)^{\frac{n}{1-a}}>1
$$

concluding the study of the quotient $\tilde{Q} / q$ near the origin.

Now, arguing by contradiction, we assume that there exists $\tilde{\lambda}>0$ such that $\tilde{Q}(\tilde{\lambda})<$ $q(\tilde{\lambda})$. By the continuity of the functions $\tilde{Q}$ and $q$, one can fix $\lambda^{\#}<\tilde{\lambda}$ to be the largest number with the property $\tilde{Q}\left(\lambda^{\#}\right)=q\left(\lambda^{\#}\right)$. Thus, $q-\tilde{Q}$ is non-negative on $\left[\lambda^{\#}, \tilde{\lambda}\right]$. We define for $\lambda>0$ the function $z_{\lambda}:(0, \infty) \longrightarrow \mathbb{R}$ by $z_{\lambda}(\rho):=C^{-2}(n-2)^{-2} \rho^{\frac{2}{p}}+\lambda \rho$. By relations (2.8) and (2.9), for every $\lambda>0$, we have

$$
z_{\lambda}\left(-\tilde{Q}^{\prime}(\lambda)\right) \leq \frac{n-1+a}{1-a} \tilde{Q}(\lambda), \quad z_{\lambda}\left(-q^{\prime}(\lambda)\right)=\frac{n-1+a}{1-a} q(\lambda) .
$$

Since $z_{\lambda}$ is increasing, one has in particular that

$$
\tilde{Q}^{\prime}(\lambda)-q^{\prime}(\lambda) \geq z_{\lambda}^{-1}\left(\frac{n-1+a}{1-a} q(\lambda)\right)-z_{\lambda}^{-1}\left(\frac{n-1+a}{1-a} \tilde{Q}(\lambda)\right), \quad \lambda \in\left[\lambda^{\#}, \tilde{\lambda}\right] .
$$

Taking into account that $z_{\lambda}^{-1}$ is increasing and $q \geq \tilde{Q}$ on $\left[\lambda^{\#}, \tilde{\lambda}\right]$, the above inequality implies that $(\tilde{Q}-q)^{\prime}(\lambda) \geq 0$ for every $\lambda \in\left[\lambda^{\#}, \tilde{\lambda}\right]$. In particular, we obtain $0>(\tilde{Q}-q)(\tilde{\lambda}) \geq$ $(\tilde{Q}-q)\left(\lambda^{\#}\right)=0$, a contradiction. This completes the proof of (2.10).

Step 4 We continue to assume $C>K_{a}$. Observe from (2.4), (2.7) and (2.10) that

$$
\int_{0}^{\infty}\left\{\mu\left(B\left(x_{0}, \rho\right)\right)-\left(C^{-1} K_{a}\right)^{\frac{n}{1-a}} \mu_{E}\left(\mathbb{B}_{n}(\rho)\right)\right\} f(\lambda, \rho) d \rho \geq 0, \quad \lambda>0 .
$$

By the hypothesis $(\mathbf{V D})_{C_{0}}^{n}$, for every $\rho>0$, we have

$$
C_{0} \frac{\mu\left(B\left(x_{0}, \rho\right)\right)}{\mu_{E}\left(\mathbb{B}_{n}(\rho)\right)} \geq \limsup _{r \rightarrow \infty} \frac{\mu\left(B\left(x_{0}, r\right)\right)}{\mu_{E}\left(\mathbb{B}_{n}(r)\right)}=: s_{0} .
$$

We claim that

$$
s_{0} \geq\left(C^{-1} K_{a}\right)^{\frac{n}{1-a}}
$$


Assuming the contrary, there exists $\delta_{0}>0$ such that, for some $r_{0}>0$,

$$
\frac{\mu\left(B\left(x_{0}, \rho\right)\right)}{\mu_{E}\left(\mathbb{B}_{n}(\rho)\right)} \leq\left(C^{-1} K_{a}\right)^{\frac{n}{1-a}}-\delta_{0} \quad \text { for all } \rho \geq r_{0}
$$

Hence, from (2.12), (2.5) and (2.4), we first have

$$
\begin{aligned}
0 \leq & \int_{0}^{\infty}\left\{\mu\left(B\left(x_{0}, \rho\right)\right)-\left(C^{-1} K_{a}\right)^{\frac{n}{1-a}} \mu_{E}\left(\mathbb{B}_{n}(\rho)\right)\right\} f(\lambda, \rho) d \rho \\
\leq & \int_{0}^{r_{0}} \mu\left(B\left(x_{0}, \rho\right)\right) f(\lambda, \rho) d \rho+\left\{\left(C^{-1} K_{a}\right)^{\frac{n}{1-a}}-\delta_{0}\right\} \int_{r_{0}}^{\infty} \mu_{E}\left(\mathbb{B}_{n}(\rho)\right) f(\lambda, \rho) d \rho \\
& -\left(C^{-1} K_{a}\right)^{\frac{n}{1-a}} \int_{0}^{\infty} \mu_{E}\left(\mathbb{B}_{n}(\rho)\right) f(\lambda, \rho) d \rho \\
\leq & C_{0} \int_{0}^{r_{0}} \mu_{E}\left(\mathbb{B}_{n}(\rho)\right) f(\lambda, \rho) d \rho-\left(C^{-1} K_{a}\right)^{\frac{n}{1-a}} \int_{0}^{r_{0}} \mu_{E}\left(\mathbb{B}_{n}(\rho)\right) f(\lambda, \rho) d \rho \\
& -\delta_{0} \int_{r_{0}}^{\infty} \mu_{E}\left(\mathbb{B}_{n}(\rho)\right) f(\lambda, \rho) d \rho \\
= & \left\{C_{0}-\left(C^{-1} K_{a}\right)^{\frac{n}{1-a}}+\delta_{0}\right\} \int_{0}^{r_{0}} \mu_{E}\left(\mathbb{B}_{n}(\rho)\right) f(\lambda, \rho) d \rho-\delta_{0} \int_{0}^{\infty} \mu_{E}\left(\mathbb{B}_{n}(\rho)\right) f(\lambda, \rho) d \rho \\
= & \left\{C_{0}-\left(C^{-1} K_{a}\right)^{\frac{n}{1-a}}+\delta_{0}\right\} \int_{0}^{r_{0}} \mu_{E}\left(\mathbb{B}_{n}(\rho)\right) f(\lambda, \rho) d \rho-\delta_{0} \frac{n-1+a}{1-a} \lambda^{\frac{n-2+2 a}{2(a-1)}} Q_{E}(1),
\end{aligned}
$$

where we used $Q_{E}(\lambda)=\lambda^{\frac{n-2+2 a}{2(a-1)}} Q_{E}(1)$ following from (2.11). Next, by using the explicit form (2.3) of $f(\lambda, \rho)$ and $a-1<0$, the following estimate holds:

$$
\begin{aligned}
\int_{0}^{r_{0}} \rho^{n} f(\lambda, \rho) d \rho & \leq \lambda^{\frac{n}{a-1}} \int_{0}^{r_{0}} \rho^{n-1-a p}\left\{\rho^{2-a p}\left(\frac{n-1+a}{1-a}(2-a p)+a p\right)+a p \lambda\right\} d \rho \\
& =\left(\frac{n-1+a}{1-a}(2-a p)+a p\right) \frac{r_{0}^{n-2 a p+2}}{n-2 a p+2} \lambda^{\frac{n}{a-1}}+a p \frac{r_{0}^{n-a p}}{n-a p} \lambda^{\frac{n}{a-1}+1} .
\end{aligned}
$$

Reorganizing the above two estimates, we obtain the inequality of type

$$
M_{1} \lambda^{\frac{n-2+2 a}{2(a-1)}} \leq M_{2} \lambda^{\frac{n}{a-1}}+M_{3} \lambda^{\frac{n}{a-1}+1} \quad \text { for all } \lambda>0
$$

where $M_{1}, M_{2}, M_{3}>0$ are constants independent of $\lambda>0$. Since

$$
\frac{n}{a-1}+1-\frac{n-2+2 a}{2(a-1)}=\frac{n}{2(a-1)}<0
$$

(2.14) fails for large values of $\lambda>0$. This contradiction shows the validity of (2.13).

Step 5 Fix any $x \in X$. Since $B\left(x_{0}, r-d\left(x_{0}, x\right)\right) \subset B(x, r) \subset B\left(x_{0}, r+d\left(x_{0}, x\right)\right)$ for every $r>d\left(x_{0}, x\right)$, on account of the hypothesis $(\mathbf{V D})_{C_{0}}^{n}$ and (2.13), one has

$$
C_{0} \frac{\mu(B(x, \rho))}{\mu_{E}\left(\mathbb{B}_{n}(\rho)\right)} \geq \limsup _{r \rightarrow \infty} \frac{\mu(B(x, r))}{\mu_{E}\left(\mathbb{B}_{n}(r)\right)}=\limsup _{r \rightarrow \infty} \frac{\mu\left(B\left(x_{0}, r\right)\right)}{\mu_{E}\left(\mathbb{B}_{n}(r)\right)}=s_{0} \geq\left(C^{-1} K_{a}\right)^{\frac{n}{1-a}}
$$

for all $\rho>0$. This concludes the proof. 


\section{Applications: Caffarelli-Kohn-Nirenberg inequal- ity on Finsler manifolds}

Before proving Theorems 1.3 and 1.4, we concisely recall some notions from the theory of Finsler manifolds (see Bao, Chern and Shen [3], Shen [15] and Ohta [10] for details), and prove the validity of the Caffarelli-Kohn-Nirenberg inequality on Minkowski spaces.

\subsection{Preliminary notions from Finsler geometry}

\subsubsection{Finsler manifolds}

Let $M$ be a connected $n$-dimensional $C^{\infty}$-manifold and $T M=\bigcup_{x \in M} T_{x} M$ be its tangent bundle.

Definition 3.1 (Finsler manifolds) The pair $(M, F)$ is called a Finsler manifold if a continuous function $F: T M \longrightarrow[0, \infty)$ satisfies the conditions:

(1) $F \in C^{\infty}(T M \backslash\{0\})$;

(2) $F(x, t v)=|t| F(x, v)$ for all $t \in \mathbb{R}$ and $(x, v) \in T M$;

(3) the $n \times n$ matrix

$$
g_{i j}(x, v):=\frac{1}{2} \frac{\partial^{2}\left(F^{2}\right)}{\partial v^{i} \partial v^{j}}(x, v), \quad \text { where } v=\sum_{i=1}^{n} v^{i} \frac{\partial}{\partial x^{i}}
$$

is positive definite for all $(x, v) \in T M \backslash\{0\}$. We will denote by $g_{v}$ the inner product on $T_{x} M$ induced from (3.1).

If (and only if) $g_{i j}(x, v)$ is independent of $v$ in each $T_{x} M \backslash\{0\}$, then $(M, F)$ gives a Riemannian manifold. A Minkowski space consists of a finite dimensional vector space $V$ and a Minkowski norm which induces a Finsler metric on $V$ by translation (i.e., $F(x, v)$ is independent of $x$ ). A Finsler manifold $(M, F)$ is called a locally Minkowski space if any point in $M$ admits a local coordinate system $\left(x^{i}\right)$ on its neighborhood such that $F(x, v)$ depends only on $v$ and not on $x$.

For a $C^{\infty}$-curve $\sigma:[0, l] \longrightarrow M$, its integral length is given by $L_{F}(\sigma):=\int_{0}^{l} F(\sigma, \dot{\sigma}) d t$. Define the distance function $d_{F}: M \times M \longrightarrow[0, \infty)$ by $d_{F}\left(x_{1}, x_{2}\right):=\inf _{\sigma} L_{F}(\sigma)$, where $\sigma$ runs over all $C^{\infty}$-curves from $x_{1}$ to $x_{2}$. When $(M, F)=\left(\mathbb{R}^{n}, F\right)$ is a Minkowski space, one has $d_{F}\left(x_{1}, x_{2}\right)=F\left(x_{2}-x_{1}\right)$. A $C^{\infty}$-curve $\sigma:[0, l] \longrightarrow M$ is called a geodesic if it is locally $d_{F}$-minimizing and has a constant speed (i.e., $F(\sigma, \dot{\sigma})$ is constant). We can write down the geodesic (Euler-Lagrange) equation in terms of the covariant derivative along $\sigma$ (see [3] for details). We say that $(M, F)$ is complete if any geodesic $\sigma:[0, l] \longrightarrow M$ can be extended to a geodesic $\sigma: \mathbb{R} \longrightarrow M$. 
The polar transform (or the dual norm) of $F$ is defined for every $(x, \alpha) \in T^{*} M$ by

$$
F^{*}(x, \alpha):=\sup _{v \in T_{x} M \backslash\{0\}} \frac{\alpha(v)}{F(x, v)} .
$$

Note that, for every $x \in M$, the function $F^{*}(x, \cdot)$ is a Minkowski norm on $T_{x}^{*} M$. In particular, if $\left(\mathbb{R}^{n}, F\right)$ is a Minkowski space, then so is $\left(\mathbb{R}^{n}, F^{*}\right)$ as well. For $u(x)=$ $d_{F}\left(x_{0}, x\right)$ with some fixed $x_{0} \in M$, one can easily see that $F^{*}(x, D u(x))=1$ for a.e. $x \in M$.

\subsubsection{Jacobi fields, Ricci curvature and volume comparison}

Let $\sigma:(-\varepsilon, \varepsilon) \times[0, l] \longrightarrow M$ be a $C^{\infty}$-geodesic variation (i.e., $t \longmapsto \sigma(s, t)$ is geodesic for each $s)$, and put $\eta(t)=\sigma(0, t)$. Then the variational vector field $J(t):=\frac{\partial \sigma}{\partial s}(0, t)$ satisfies the Jacobi equation

$$
D_{\dot{\eta}}^{\dot{\eta}} D_{\dot{\eta}}^{\dot{\eta}} J+R^{\dot{\eta}}(J, \dot{\eta}) \dot{\eta} \equiv 0
$$

where $D^{\dot{\eta}}$ is the covariant derivative with reference vector $\dot{\eta}$, and $R^{\dot{\eta}}$ is the curvature tensor (see [3] for details). For two linearly independent vectors $v, w \in T_{x} M$ and $\mathcal{S}=\operatorname{span}\{v, w\}$, the flag curvature of the flag $(\mathcal{S} ; v)$ is defined by

$$
K(\mathcal{S} ; v):=\frac{g_{v}\left(R^{v}(w, v) v, w\right)}{F(v)^{2} g_{v}(w, w)-g_{v}(v, w)^{2}} .
$$

If $(M, F)$ is Riemannian, then the flag curvature reduces to the sectional curvature which depends only on $\mathcal{S}$ (not on the choice of $v \in \mathcal{S}$ ). Take $v \in T_{x} M$ with $F(x, v)=1$ and let $\left\{e_{i}\right\}_{i=1}^{n}$ with $e_{n}=v$ be an orthonormal basis of $\left(T_{x} M, g_{v}\right)$ for $g_{v}$ from (3.1). Put $\mathcal{S}_{i}=\operatorname{span}\left\{e_{i}, v\right\}$ for $i=1, \ldots, n-1$. Then the Ricci curvature of $v$ is defined by $\operatorname{Ric}(v):=\sum_{i=1}^{n-1} K\left(\mathcal{S}_{i} ; v\right)$. For $c \geq 0$, we also set $\operatorname{Ric}(c v):=c^{2} \operatorname{Ric}(v)$.

Shen gave a useful interpretation of these Finsler curvatures from the Riemannian viewpoint (see [15, §6.2]). Fix $v \in T_{x} M \backslash\{0\}$ and extend it to a $C^{\infty}$-vector field $V$ around $x$ such that all integral curves of $V$ are geodesic. Then the flag curvature $K(\mathcal{S} ; v)$ coincides with the sectional curvature of $\mathcal{S}$ with respect to the Riemannian structure $g_{V}$, and $\operatorname{Ric}(v)$ coincides with the Ricci curvature of $v$ with respect to $g_{V}$. This observation leads the following definition of the weighted Ricci curvature associated with an arbitrary measure on $M$. We refer to [10], [12, [13], and [11] for details and applications.

Definition 3.2 (Weighted Ricci curvature) Let $\mu$ be a positive $C^{\infty}$-measure on $M$. Given $v \in T_{x} M \backslash\{0\}$, let $\sigma:(-\varepsilon, \varepsilon) \longrightarrow M$ be the geodesic with $\dot{\sigma}(0)=v$ and decompose $\mu$ along $\sigma$ as $\mu=e^{-\psi} \operatorname{vol}_{\dot{\sigma}}$, where $\operatorname{vol}_{\dot{\sigma}}$ denotes the volume form of the Riemannian structure $g_{\dot{\sigma}}$. Then, for $N \in[n, \infty]$, the $N$-Ricci curvature $\operatorname{Ric}_{N}$ is defined by

$$
\operatorname{Ric}_{N}(v):=\operatorname{Ric}(v)+(\psi \circ \sigma)^{\prime \prime}(0)-\frac{(\psi \circ \sigma)^{\prime}(0)^{2}}{N-n},
$$

where the third term is understood as 0 if $N=\infty$ or if $N=n$ with $(\psi \circ \sigma)^{\prime}(0)=0$, and as $-\infty$ if $N=n$ with $(\psi \circ \sigma)^{\prime}(0) \neq 0$. 
In particular, $\operatorname{Ric}_{n}$ is bounded below only if $(\psi \circ \sigma)^{\prime} \equiv 0$ along any $\sigma$. In terms of $\mathrm{Ric}_{N}$, one can show the following Bishop-Gromov-type volume comparison theorem. (Indeed, we can reduce it to the Riemannian setting by using the gradient vector field of the distance function from the center $x$.) We state only the non-negatively curved case.

Theorem 3.3 ([10, Theorem 7.3]) Let $(M, F, \mu)$ be a complete $n$-dimensional Finsler manifold with non-negative $N$-Ricci curvature. Then we have

$$
\frac{\mu(B(x, R))}{\mu(B(x, r))} \leq\left(\frac{R}{r}\right)^{N} \quad \text { for every } x \in M, 0<r<R .
$$

Moreover, if equality holds with $N=n$ for all $x \in M$ and $0<r<R$, then any Jacobi field $J$ along a geodesic $\sigma$ has the form $J(t)=t P(t)$, where $P$ is a parallel vector field along $\sigma$ (i.e., $\left.D_{\dot{\sigma}}^{\dot{\sigma}} P \equiv 0\right)$.

We will actually need only the most restrictive case of $N=n$.

\subsection{Caffarelli-Kohn-Nirenberg inequality on Minkowski spaces}

Let $(M, F)$ be a Finsler manifold and $u \in \operatorname{Lip}_{0}(M)$. Note that the local Lipschitz constant of $u$ is given by $|D u|(x)=F^{*}(x, D u(x))$ for a.e. $x \in M$. Therefore, due to density reasons, the Caffarelli-Kohn-Nirenberg inequality $(\mathbf{C K N})_{C}^{x_{0}}$ in the Finsler context takes the more familiar form

$$
\left(\int_{M} \frac{|u(x)|^{p}}{d_{F}\left(x_{0}, x\right)^{a p}} d \mu(x)\right)^{\frac{1}{p}} \leq C\left(\int_{M} F^{*}(x, D u(x))^{2} d \mu(x)\right)^{\frac{1}{2}} \quad \text { for all } u \in C_{0}^{\infty}(M) .
$$

We first prove that the sharp Caffarelli-Kohn-Nirenberg inequality (with $C=K_{a}$ from (1.1) ) holds on an arbitrary Minkowski space $\left(\mathbb{R}^{n}, F\right)$ endowed with the Lebesgue measure $\mu_{F}$ normalized so that $\mu_{F}(B(0,1))=\omega_{n}$.

Let us first recall two inequalities on $\left(\mathbb{R}^{n}, F, \mu_{F}\right)$. Given a measurable set $\Omega \subset \mathbb{R}^{n}$, let us denote by $\Omega^{\star}$ the anisotropic symmetrization of $\Omega$, i.e., it is the open ball with center 0 such that $\mu_{F}\left(\Omega^{\star}\right)=\mu_{F}(\Omega)$. For a function $u: \mathbb{R}^{n} \longrightarrow \mathbb{R}, u^{\star}(x):=\sup \left\{c \in \mathbb{R}: x \in\{u>c\}^{\star}\right\}$ is the anisotropic (decreasing) symmetrization of $u$, where $\{u>c\}=\left\{x \in \mathbb{R}^{n}: u(x)>c\right\}$. Due to Alvino, Ferone, Lions and Trombetti [1] and Van Schaftingen [18], one has

- anisotropic Pólya-Szegő inequality:

$$
\int_{\mathbb{R}^{n}} F^{*}\left(D u^{\star}(x)\right)^{2} d \mu_{F}(x) \leq \int_{\mathbb{R}^{n}} F^{*}(D u(x))^{2} d \mu_{F}(x) \quad \text { for all } u \in C_{0}^{\infty}\left(\mathbb{R}^{n}\right)_{+} ;
$$

- anisotropic Hardy-Littlewood inequality: if $p>1$ and $a \in[0,1]$, then we have

$$
\int_{\mathbb{R}^{n}} \frac{u(x)^{p}}{F(x)^{a p}} d \mu_{F}(x) \leq \int_{\mathbb{R}^{n}} \frac{u^{\star}(x)^{p}}{F(x)^{a p}} d \mu_{F}(x) \text { for all } u \in C_{0}^{\infty}\left(\mathbb{R}^{n}\right)_{+},
$$

where $C_{0}^{\infty}\left(\mathbb{R}^{n}\right)_{+}:=\left\{u \in C_{0}^{\infty}\left(\mathbb{R}^{n}\right): u \geq 0\right\}$. 
Proposition 3.4 Let $\left(\mathbb{R}^{n}, F\right)$ be a Minkowski space with $n \geq 3, x_{0} \in \mathbb{R}^{n}, a \in[0,1)$, and $p=2 n /(n-2+2 a)$. Then the sharp Caffarelli-Kohn-Nirenberg inequality $(\mathbf{C K N})_{K_{a}}^{x_{0}}$ holds on $\left(\mathbb{R}^{n}, F, \mu_{F}\right)$. Moreover, the constant $K_{a}$ is optimal and a family of extremals is given by

$$
u_{\lambda}(x)=\left(\lambda+F\left(x-x_{0}\right)^{2-a p}\right)^{\frac{2-n}{2-a p}}, \quad \lambda>0 .
$$

Proof. Recall that $d_{F}\left(x_{0}, x\right)=F\left(x-x_{0}\right)$. Without loss of generality, we may assume that $x_{0}=0$. Let us consider the constant

$$
C_{a}=\inf _{u \in C_{0}^{\infty}\left(\mathbb{R}^{n}\right) \backslash\{0\}} \frac{\left(\int_{\mathbb{R}^{n}} F^{*}(D u(x))^{2} d \mu_{F}(x)\right)^{1 / 2}}{\left(\int_{\mathbb{R}^{n}}|u(x)|^{p} F(x)^{-a p} d \mu_{F}(x)\right)^{1 / p}} .
$$

We shall claim that $C_{a}=K_{a}^{-1}$. Due to the reversibility of $F$, it is enough to consider nonnegative functions in the above expression. By the anisotropic Pólya-Szegó and HardyLittlewood inequalities we have

$$
C_{a}=\inf _{u \in C_{0}^{\infty}\left(\mathbb{R}^{n}\right)_{+} \backslash\{0\}} \frac{\left(\int_{\mathbb{R}^{n}} F^{*}\left(D u^{\star}(x)\right)^{2} d \mu_{F}(x)\right)^{1 / 2}}{\left(\int_{\mathbb{R}^{n}} u^{\star}(x)^{p} F(x)^{-a p} d \mu_{F}(x)\right)^{1 / p}} .
$$

We may assume that $u^{\star} \in C_{0}^{1}\left(\mathbb{R}^{n}\right)_{+}$(otherwise, a density argument applies). Then there exists a non-increasing function $h:[0, \infty) \longrightarrow[0, \infty)$ of class $C^{1}$ such that $u^{\star}(x)=$ $h(F(x))$, and we have

$$
F^{*}\left(D u^{\star}(x)\right)=F^{*}\left(h^{\prime}(F(x)) D F(x)\right)=-h^{\prime}(F(x)) F^{*}(D F(x))=-h^{\prime}(F(x)) .
$$

Therefore a simple calculation yields

$$
\frac{\left(\int_{\mathbb{R}^{n}} F^{* 2}\left(D u^{\star}(x)\right) d \mu_{F}(x)\right)^{1 / 2}}{\left(\int_{\mathbb{R}^{n}} u^{\star}(x)^{p} F(x)^{-a p} d \mu_{F}(x)\right)^{1 / p}}=\alpha_{n}^{\frac{1}{2}-\frac{1}{p}} \frac{\left(\int_{0}^{\infty} h^{\prime}(\rho)^{2} \rho^{n-1} d \rho\right)^{1 / 2}}{\left(\int_{0}^{\infty} h(\rho)^{p} \rho^{n-1-a p} d \rho\right)^{1 / p}},
$$

where $\alpha_{n}=n \omega_{n}$ denotes the area of the unit sphere in $\mathbb{R}^{n}$. On the other hand, following the approaches of Lieb [9] and Talenti [17] in the Euclidean case where the standard Schwarz symmetrization is used, one can see that the minimizing expression is precisely the RHS of (3.3) . Therefore, we have $C_{a}=K_{a}^{-1}$ which proves our claim. Moreover, a class of minimizers $h_{\lambda}$ for (3.3) is $h_{\lambda}(\rho)=\left(\lambda+\rho^{2-a p}\right)^{\frac{2-n}{2-a p}}, \lambda>0$, which can be obtained by the standard Euler-Lagrange method.

Remark 3.5 After a slight modification, Proposition 3.4 remains valid also for only positively homogeneous Minkowski norms (i.e., $F(t v)=t F(v)$ only for $t>0$ ). In such a case, the anisotropic symmetrization is considered with respect to the backward ball $B_{-}(0,1)=\left\{x \in \mathbb{R}^{n}: F(-x)<1\right\}$, and the level sets of the extremals have backward Wulff-shapes, homothetic to $B_{-}(0,1)$ (see Kristály [8] and Van Shaftingen [18]). 


\subsection{Proof of Theorems 1.3 and 1.4}

Proof of Theorem 1.3. Since $(M, F)$ is complete, by the Hopf-Rinow theorem it yields that $\left(M, d_{F}, \mu\right)$ is a proper metric measure space. On account of Theorem 3.3 , (VD) ${ }_{C_{0}}^{n}$ holds with $C_{0}=1$, while $\mu$ is normalized so as to satisfy $(\mathbf{A R})_{x_{0}}^{n}$. On the one hand, these properties imply that

$$
\mu(B(x, \rho)) \leq \mu_{E}\left(\mathbb{B}_{n}(\rho)\right) \text { for all } \rho>0, x \in M .
$$

On the other hand, by $(\mathbf{C K N})_{K_{a}}^{x_{0}}$, Theorem 1.1 gives the reverse inequality, thus equality holds. By Theorem 3.3, it results that every Jacobi field $J$ along any geodesic $\sigma$ has the form $J(t)=t P(t)$, where $P$ is a parallel vector field along $\sigma$. Then it follows from the Jacobi equation (3.2) that $R^{\dot{\sigma}}(J, \dot{\sigma}) \dot{\sigma} \equiv 0$, so that $K(\mathcal{S} ; \dot{\sigma}) \equiv 0$ with $\mathcal{S}=\operatorname{span}\{\dot{\sigma}, P\}$. Due to the arbitrariness of $\sigma$ and $J$, it turns out that the flag curvature of $(M, F)$ is identically zero.

Proof of Theorem 1.4. On the one hand, since on every Berwald space Ric $_{n}=$ Ric holds for the Hausdorff measure $\mu_{F}$ (see Shen [14, Propositions $\left.2.6 \& 2.7\right]$ ), we can apply Theorem 1.3 to see that the flag curvature of $(M, F)$ is identically zero. On the other hand, every Berwald space with zero flag curvature is necessarily a locally Minkowski space (see Bao, Chern and Shen [3, Section 10.5]). Thanks to the volume identity $\mu_{F}(B(x, \rho))=\mu_{E}\left(\mathbb{B}_{n}(\rho)\right),(M, F)$ must be isometric to a Minkowski space.

We conclude the paper by presenting an example of a non-Riemannian Berwald space.

Example 3.6 We endow the space $\mathbb{R}^{n-1}(n \geq 3)$ with a Riemannian metric $g$ such that $\left(\mathbb{R}^{n-1}, g\right)$ is complete with non-negative Ricci curvature. For every $\varepsilon>0$, consider on $\mathbb{R}^{n}=\mathbb{R}^{n-1} \times \mathbb{R}$ the metric $F_{\varepsilon}: T \mathbb{R}^{n} \longrightarrow[0, \infty)$ given by

$$
F_{\varepsilon}((x, t),(v, w))=\sqrt{g_{x}(v, v)+w^{2}+\varepsilon \sqrt{g_{x}(v, v)^{2}+w^{4}}}
$$

for $(x, t) \in \mathbb{R}^{n},(v, w) \in T_{x} \mathbb{R}^{n-1} \times T_{t} \mathbb{R}$. We observe that $\left(\mathbb{R}^{n}, F_{\varepsilon}\right)$ is a non-compact, complete, non-Riemannian Berwald space with non-negative Ricci curvature. According to Theorem 1.4 and Proposition 3.4, the following four statements are equivalent:

- $(\mathbf{C K N})_{K_{a}}^{\tilde{x}_{0}}$ holds on $\left(\mathbb{R}^{n}, F_{\varepsilon}, \mu_{F_{\varepsilon}}\right)$ for some element $\tilde{x}_{0}=\left(x_{0}, t_{0}\right) \in \mathbb{R}^{n}$;

- $(\mathbf{C K N})_{K_{a}}^{\tilde{x}_{0}}$ holds on $\left(\mathbb{R}^{n}, F_{\varepsilon}, \mu_{F_{\varepsilon}}\right)$ for every element $\tilde{x}_{0}=\left(x_{0}, t_{0}\right) \in \mathbb{R}^{n}$;

- $g_{x}$ is independent of $x \in \mathbb{R}^{n-1}$ (i.e., $\left(\mathbb{R}^{n-1}, g\right)$ is flat);

- $\left(\mathbb{R}^{n}, F_{\varepsilon}\right)$ is a Minkowski space.

Acknowledgment. The paper was completed when A. Kristály visited the Department of Mathematics of Kyoto University in October 2012. He is supported by a grant of the Romanian National Authority for Scientific Research, CNCS-UEFISCDI, project number PN-II-ID-PCE-2011-3-0241 and partially by Domus Hungarica. S. Ohta is supported by the Grant-in-Aid for Young Scientists (B) 23740048. 


\section{References}

[1] A. Alvino, V. Ferone, P.-L. Lions, G. Trombetti, Convex symmetrization and applications. Ann. Inst. H. Poincaré Anal. Non Linéaire 14 (1997), no. 2, 275-293.

[2] T. Aubin, Problèmes isopérimétriques de Sobolev. J. Differ. Geom. 11 (1976), 573598.

[3] D. Bao, S.-S. Chern, Z. Shen, Introduction to Riemann-Finsler Geometry, Graduate Texts in Mathematics, 200, Springer Verlag, 2000.

[4] L. Caffarelli, R. Kohn, L. Nirenberg, First order interpolation inequalities with weight. Compos. Math. 53 (1984), 259-275.

[5] K. S. Chou, C. W. Chu, On the best constant for a weighted Sobolev-Hardy inequality. J. London Math. Soc. (2) 48 (1993), no. 1, 137-151.

[6] M. P. do Carmo, C. Xia, Complete manifolds with non-negative Ricci curvature and the Caffarelli-Kohn-Nirenberg inequalities. Compos. Math. 140 (2004), 818-826.

[7] J. Heinonen, Lectures on Analysis on Metric Spaces, Springer, New York, 2001.

[8] A. Kristály, Anisotropic singular phenomena in the presence of asymmetric Minkowski norms. Preprint (2012).

[9] E. H. Lieb, Sharp constants in the Hardy-Littlewood and related inequalities. Ann. of Math. (2) 118 (1983), 349-374.

[10] S. Ohta, Finsler interpolation inequalities. Calc. Var. Partial Differential Equations 36 (2009), 211-249.

[11] S. Ohta, Splitting theorems for Finsler manifolds of nonnegative Ricci curvature. J. Reine Angew. Math. (to appear). Available at arXiv:1203.0079

[12] S. Ohta, K.-T. Sturm, Heat flow on Finsler manifolds. Comm. Pure Appl. Math. 62 (2009), no. 10, 1386-1433.

[13] S. Ohta, K.-T. Sturm, Bochner-Weitzenböck formula and Li-Yau estimates on Finsler manifolds. Preprint (2011). Available at arXiv:1104.5276

[14] Z. Shen, Volume comparison and its applications in Riemann-Finsler geometry. Adv. Math. 128 (1997), no. 2, 306-328.

[15] Z. Shen, Lectures on Finsler geometry, World Scientific Publishing Co., Singapore, 2001.

[16] Z. I. Szabó, Positive definite Berwald spaces. Structure theorems on Berwald spaces. Tensor (N.S.) 35 (1981), no. 1, 25-39. 
[17] G. Talenti, Best constant in Sobolev inequality. Ann. Mat. Pura Appl. (4) 110 (1976), 353-372.

[18] J. Van Schaftingen, Anisotropic symmetrization. Ann. Inst. H. Poincaré Anal. Non Linéaire 23 (2006), no. 4, 539-565. 